\title{
Enfoque holístico de la metodología de los Estilos de Aprendizaje en una institución educativa
}

\author{
Holistic approach to the methodology of Learning \\ Styles in an educational institution
}

DOMINGOJ. GALLEGO GIL(iDa

Catalina M. Alonso id b

\section{Resumen}

En este trabajo pretendemos facilitar un enfoque holístico, completo y riguroso, sobre la Metodología de los Estilos de Aprendizaje en el que participe toda la Comunidad Educativa. Después de preguntarnos por qué la Metodología de los Estilos de Aprendizaje sigue sin incluirse en los curricula, tratamos de superar el individualismo investigador e innovador para integrarlo en una dinámica en la que participen todos los miembros de la Comunidad Educativa. Se propone una decisión y un compromiso del equipo directivo del Centro que asuma la oportunidad e interés de introducir la metodología de los Estilos de Aprendizaje en nuestro diseño educativo de forma planificada y coordinada en todas las aulas del Centro. Para ello hace falta tener en cuenta los aspectos y pasos que describimos detalladamente en tres etapas: Diseñar, Desarrollar y Evaluar/Supervisar.

Palabras clave: Estilos de Aprendizaje. Innovación educativa. Proyecto de Centro. Dirección de Centros.

\footnotetext{
Abstract

In this work we intend to facilitate a holistic approach, complete and rigorous, on the Methodology of Learning Styles in which the entire Educational Community participates. After asking ourselves why the Methodology of Learning Styles is still not

a Universidad Nacional de Educación a Distancia, UNED, Madrid, España. Doctor en Filosofía y Letras, domingoj.gallego@gmail.com

b Universidad Nacional de Educación a Distancia, UNED, Madrid, España. en Filosofía y Ciencias de la Educación, catalinam.alonso@gmail.com
} 
included in the curricula, we try to overcome research and innovative individualism to integrate it into a dynamic in which all members of the Educational Community participate. It is proposed a decision and a commitment of the management team of the Center that assumes the opportunity and interest to introduce the methodology of Learning Styles in our educational design in a planned and coordinated manner in all classrooms of the Center. This requires taking into account the aspects and steps that we describe in detail in three stages: Design, Develop and Evaluate / Supervise.

Keywords: Learning Styles. Educational innovation. Center project. Institution Management.

\section{Punto de Partida}

A lo largo de los años hemos impartido muchas jornadas de diagnóstico y aplicaciones prácticas de los Estilos de Aprendizaje a estudiantes y a profesores en ejercicio. Estos Encuentros siempre han finalizado con la satisfacción de los participantes y comentarios de que les ha resultado muy interesante y útil el enfoque metodológico de los Estilos de Aprendizaje, lamentando que no se les hubiera presentado antes esta temática. Sin embargo, en la mayoría de los curricula de Pedagogía, Psicopedagogía, Educación Social, en las Universidades Españolas o en los planes de Formación Permanente del profesorado sigue sin incluirse un apartado explícito referente al diagnóstico y aplicaciones de los Estilos de Aprendizaje.

Nos hemos preguntado muchas veces el porqué de este dato, que nos parece muy poco positivo. Una primera respuesta indica que la pluralidad de taxonomías, clasificaciones y herramientas de diagnóstico se ha convertido en una barrera práctica para la toma de decisiones y la elección concreta de un camino de acción. J. L. García Cué, J. A. Santizo y C. M. Alonso (2009) hacen un excelente resumen resaltando, entre más de cien herramientas, los 38 instrumentos de diagnóstico más utilizados para la metodología los Estilos de Aprendizaje.

Una segunda razón la atribuimos a la herramienta $V A K$, que ha alcanzado popularidad, se centra exclusivamente en las diferencias perceptivas de los individuos para presentar tres estilos de aprendizaje visual, auditivo y kinésico (se convierte en cuatro estilos en formatos posteriores denominados VARK). Esta clasificación ha resultado superficial y tampoco ha sido una ayuda para el 
profesorado, sino más bien el blanco preferido de los críticos contra los Estilos de Aprendizaje. Sharp, J. G., Bowker, R., \& Byrne, J. (2008), Gholami, S., \& Bagheri, M. (2013).

En tercer lugar, destacamos la oposición a los Estilos de Aprendizaje de algunos autores. Desde que Pashler, H., McDaniel, M., Rohrer, D., \& Bjork, R. (2009) y Riener, C. \& Willingham, D. (2010) calificaran los Estilos de Aprendizaje como "mito", hemos accedido a varios artículos críticos posteriores Fleming, N. D. (2012), Hussman, P., \& Dean O’Loughlin, V., (2018) Jena, A. K., \& Chakraborty, S. (2018), Kirschner, P. (2017). Li, Y., Medwell, J., Wray, D., Wang, L., \& Liu, X. (2016), Newton, P. (2015) que repiten los mismos argumentos contra los Estilos de Aprendizaje. Solo conocen la propuesta VAK y contra ella descargan sus argumentos, ignorando las otras taxonomías. Afirman que "no existe" investigación" sobre los Estilos de Aprendizaje ignorando todos los trabajos que no están redactados en lengua inglesa e ignorando también los trabajos en inglés que desde los años 60 son accesibles, muchos de ellos también recogidos en la Bibliografía presentada por C. M. Alonso en su tesis doctoral (1992). Hoy, solamente analizando los datos de ERIC, Educational Resources Information Center, se indican, desde el año 2001, 10.284 trabajos e investigaciones sobre Estilos de Aprendizaje. Si añadimos los datos ofrecidos por Dialnet que reseña 446 trabajos sobre el tema, la Revista de Estilos de Aprendizaje 240 artículos, Teseo 62 tesis doctorales, está claramente demostrado el volumen investigador sobre Estilos de Aprendizaje y su importancia práctica en los diseños de enseñanza-aprendizaje.

Es importante destacar qué es lo que se pretende cuando utilizamos la metodología de los Estilos de Aprendizaje en las investigaciones y en las experiencias. Hemos encontrado seis respuestas más frecuentes a nuestra pregunta (hay otras). Se busca la innovación educativa, mejorar la calidad educativa, integrar la tecnología para el aprendizaje, mayor eficacia y eficiencia en el aprendizaje, orientación educativa, mejorar índice de abandono escolar. Según el objetivo pretendido se han elegido herramientas de diagnóstico y estrategias de aplicación. Gokalp, M. (2013), Gappi, L. L. (2013), Altun, H. (2019), Alnujaidi, S. (2019), Biabani, M., Izadpanah, S. (2019). 
Podemos diferenciar los trabajos sobre Estilos de Aprendizaje teniendo en cuenta los niveles educativos en los que se centran. Se refieren a educación infantil, primaria, secundaria, profesional, universitaria, permanente, formación de empresa, tercera edad... Encontramos investigaciones y experiencias que aplican la metodología de los Estilos de Aprendizaje en todos los niveles educativos, por su flexibilidad y posibilidades adaptativas. Fernandes, A. A. (2011), Wininger, St. R.; Redifer, J. L.; Norman, A. D.; Ryle, M. K. (2019), Günes, G., Sahin, V. (2019), Kamisli, H.; Özonur, M. (2019).

Constatamos también la amplia variedad de temas y contenidos en los que se han aplicado y aplican los Estilos de Aprendizaje: Matemáticas, farmacia, física, economía, ciencias, estudio de lenguas, comparaciones entre estudiantes de diferentes nacionalidades, estudiantes con problemas de aprendizaje, orientación vocacional, prevención del fracaso escolar, libros de texto, PNL, inteligencias múltiples, hemisferios cerebrales, TIC, rendimiento académico, elearning, competencias, padres de estudiantes, inteligencia emocional, aprendizaje significativo, tiempo libre y ocio, objetos de aprendizaje... Sener, S., \& Cokcaliskan, A. (2018) Soiferman, K. (2019), Aslaksen, K.; Loras, H. (2019), Edwards, D. J.; Kupczynski, L.; Groff, S. (2019)

Abordamos una nueva respuesta: identificamos un cierto individualismo o particularismo innovador, pero independiente y aislado. Evidentemente son muchos los temas y muchas las propuestas educativas que se han ido estudiando, sin embargo, en casi todos los trabajos encontramos sobre todo un profesor o un grupo de profesores entusiasta y dedicado. Son docentes decididos a la innovación educativa y a buscar la mejora en los procesos de enseñanza-aprendizaje de sus estudiantes. Algunos han realizado un diagnóstico de los Estilos de Aprendizaje de sus alumnos y luego tratan de llevar adelante las conclusiones del diagnóstico. Pero suelen ser acciones personales, individuales, de un profesor que, aunque son eficaces en su aula de clase, no tienen una repercusión en otras aulas, ni una coordinación con otros docentes, se incluyen en las líneas de los proyectos de aula, pero no en los proyectos del Centro educativo.

En este trabajo pretendemos facilitar un enfoque holístico, más completo y riguroso, en el que participe toda la comunidad educativa. Superar el individualismo 
investigador e innovador para integrarlo en una dinámica en la que participen todos. Se propone una decisión del equipo directivo del Centro que asuma la oportunidad e interés de introducir la metodología de los Estilos de Aprendizaje de forma planificada y coordinada en todas las aulas del Centro. ¿Cómo afrontar esta propuesta? ¿Cómo diseñar, desarrollar y supervisar la metodología de los Estilos de Aprendizaje para que participen el equipo directivo, todos los profesores, los alumnos, los padres de los estudiantes y se incluya en el proyecto general del Centro? ¿Qué estrategia conviene seguir? ¿Cómo motivar y presentar esta propuesta a profesores y padres? ¿Cómo implementarla? ¿Cómo supervisarla? Estas son las preguntas que intentamos responder en este trabajo.

Nuestra propuesta se incluye dentro de la línea investigación-acción, investigamos para lo que hacemos lo hagamos mejor. La estrategia que sugerimos es incluir la metodología de los Estilos de Aprendizaje en nuestro diseño educativo y proyecto de Centro, para ello hace falta tener en cuenta los aspectos y pasos que debemos recorrer.

\section{La metodología de los Estilos de Aprendizaje en nuestro diseño educativo y proyecto de Centro}

La propuesta va a tener tres grandes etapas: Diseñar, Desarrollar, EvaluarSupervisar, que vamos a detallar a continuación.

Diseñar

Todo plan de acción debe contar con un diseño detallado y participativo por parte de toda la comunidad educativa liderada por el equipo directivo del Centro.

El primer paso es el análisis y el estudio del concepto y aplicaciones de los Estilos de Aprendizaje por parte del equipo directivo del Centro. Este análisis y el convencimiento de que podemos mejorar el nivel de calidad de los procesos de enseñanza-aprendizaje del Centro incorporando la metodología de los Estilos de Aprendizaje es la base sobre la que se van a desarrollar las etapas posteriores. Hay que analizar y elegir los instrumentos de diagnóstico que se van a emplear en el proceso. De una elección acertada va a depender el proceso. 
Segundo paso: El equipo directivo debe autodiagnosticar sus Estilos de Aprendizaje, aplicándose las herramientas de diagnóstico seleccionadas y comentar en una reunión específica del equipo, los resultados obtenidos. Una experiencia personal de diagnóstico y reflexión que construya un convencimiento del equipo directivo acerca de las posibilidades y ventajas de esta metodología, nos parece imprescindible para garantizar el diseño e implementación de esta innovación educativa en todas las aulas del Centro. En esta reunión se deben planificar los pasos a llevar a cabo y la temporalización de cada uno.

Tercer paso: Una vez que el equipo directivo está convencido de las posibilidades educativas de los Estilos de Aprendizaje, conviene entablar un diálogo "informal" con los profesores del Centro, más cercanos al equipo directivo y a la metodología de los Estilos de Aprendizaje, comentando con ellos la propuesta de tener una reunión con todos los docentes para presentar este plan de acción. Es importante contar desde el principio con el apoyo de docentes animados y favorables que puedan también ir comentando con sus compañeros la propuesta que se prepara, para que el "ambiente" sea propicio y receptivo a las propuestas de innovación educativa en la que van a participar todos los docentes. Se trata de un "proceso de sensibilización” muy importante en el que se necesitan todos los apoyos posibles.

El cuarto paso es la presentación a los profesores del Centro, del interés y conveniencia de incluir en la actividad docente la metodología de los Estilos de Aprendizaje. Aunque la mayoría de los docentes creen que entienden el significado de Estilos de Aprendizaje, con frecuencia tienen diferentes conceptualizaciones que conviene aclarar y precisar. A veces los profesores se sienten sobrecargados de trabajo y temen comenzar nuevas iniciativas que les compliquen su actividad diaria. Por eso se trata de convencer y motivar a los docentes de las ventajas de la metodología de los Estilos de Aprendizaje para mejorar los procesos de enseñanza/aprendizaje, superando posibles miedos y dudas. Presentamos una propuesta para todo el Centro en la que van a participar todos los profesores y en todas las aulas. Se va a facilitar formación para llevar a cabo el diagnóstico de los docentes y discentes y para desarrollar las aplicaciones prácticas de los resultados. Debe ser una reunión abierta que permita las preguntas y el diálogo. 
Destacamos algunas cuestiones útiles que deben plantearse antes de la sesión de formación que se va a realizar para conocer el pensamiento de los profesores ante esta innovación educativa. Se puede abrir un diálogo con preguntas de este tipo: ¿Qué conoce acerca de los Estilos de Aprendizaje? ¿Cuál es su nivel de interés en intentar estrategias de aplicación de los Estilos de Aprendizaje? (calificar en una escala Likert de 1 a 5) ¿Le interesa identificar su Estilo de Aprendizaje? ¿Le gustaría participar en una sesión de formación sobre los Estilos de Aprendizaje?

Una vez aceptado el plan por parte de los profesores, hay que presentar a los padres esta propuesta. Se comienza por una reunión con la asociación de padres o con los representantes de los padres en el Centro en la que se explicará la metodología de los Estilos de Aprendizaje y cómo se realizan los diagnósticos y cómo se adapta la acción docente a la peculiaridad de cada estudiante. Suele ser efectivo experimentar con un autodiagnóstico de los Estilos de Aprendizaje de los padres, utilizando la herramienta elegida, para que entiendan cómo cada niño tiene un estilo o estilos de aprendizaje preferidos y lo que a los padres les funciona bien, por su propio estilo, puede ser que a los niños no les resulte adecuado, ya que pueden tener estilos de aprendizaje muy diferentes. Con frecuencia los padres tratan de sugerir a sus hijos sus propias estrategias de aprendizaje en casa que a veces no son las más apropiadas, pues responden más a las preferencias de su estilo de aprendizaje que al estilo de aprendizaje de su hijo. Una vez conseguido el apoyo de los representantes de los padres conviene convocar una reunión con todos los padres, que puedan asistir, para explicar y compartir con todos lo que significa la metodología de los Estilos de Aprendizaje y cómo se va a ir aplicando en el Centro.

Una iniciativa de este tipo necesita que dediquemos un tiempo a la formación de los profesores en dos etapas. Se comienza por crear un grupo de profesores y capacitarlos en la tecnología del diagnóstico, interpretación de resultados, aplicación de los resultados a los casos concretos y a las peculiaridades de cada estudiante. Este grupo va a actuar como asesores de Estilos de Aprendizaje. Para impartir esta formación habrá que acudir a expertos internos o externos en Estilos de Aprendizaje, que completen tanto la labor de motivación para llevar a cabo esta tarea como la explicación del concepto de Estilos de Aprendizaje con ejemplos y propuestas variadas para que puedan aplicarse con los alumnos. Es importante la 
presencia del equipo directivo en esta formación, no sólo para demostrar el máximo interés y compromiso, sino también para formarse ellos, como asesores de Estilos de Aprendizaje.

Cuando el grupo de asesores esté preparado para ayudar a formar a sus compañeros y a los padres de los alumnos en la metodología de los Estilos de Aprendizaje, con una o más jornadas de formación, podemos seguir avanzando. El grupo de asesores facilitará a sus compañeros la capacitación para poner en marcha el proceso de innovación y va a servir de apoyo y consulta para las dudas que puedan surgir durante los procesos de implementación. Cada docente y cada padre que participe, debe contar con un documento escrito o digitalizado en la web que recoja los aspectos más importantes de la capacitación, para que pueda revisarlos cuando lo necesite, y sugerencias prácticas concretas para aplicar, tanto en el aula como en casa, la metodología de los Estilos de Aprendizaje, propuestas de cómo mejorar los Estilos de Aprendizaje, cómo superar los posibles bloqueos en formas de aprender, cómo aplicar esta metodología a los niños que necesitan ayuda para el aprendizaje (educación inclusiva) o a los alumnos de alta capacidad. En esta etapa se concreta según la edad de los estudiantes o nivel educativo la forma de hacer el diagnóstico, y cómo se interpretan los datos obtenidos y el calendario de aplicación.

\section{Desarrollar}

Después de la preparación detallada, que hemos descrito en el epígrafe Diseño, se trata ahora de desarrollar el proyecto en el que debe intervenir, como en todo el proceso, toda la comunidad educativa.

Una vez que los profesores están formados, y los padres informados, se procede a realizar el diagnóstico de los estudiantes. Se comienza el proceso de diagnóstico por algunas aulas seleccionadas, conformarán un grupo experimental, con los profesores más preparados y con el apoyo directo de los asesores. Tendremos así un grupo piloto de aulas que experimentarán el proceso y ayudarán con su acción a la resolución de problemas y a la mejora del mismo.

El proceso de diagnóstico termina con una reunión de análisis de resultados en la que participa el profesor de aula, el tutor y el, o los asesores. En esta sesión hay que estudiar el proceso de diagnóstico y las dificultades encontradas para que no se 
repitan y dificulten el proceso de diagnóstico en otras aulas. Se analizan los datos de cada alumno, sus peculiaridades, sus preferencias y sus dificultades de aprendizaje. Hay que tener muy en cuenta las aportaciones que cada profesor y tutor pueden y deben hacer con su observación directa de la conducta de los alumnos en el aula. Tras este análisis se podrá diseñar un plan de acción concreto para el docente (que deberá incorporar en su enseñanza) y para cada estudiante, según sus características. Los datos obtenidos y el plan de acción pueden comentarse con los padres de los estudiantes de cada aula en una reunión con el profesor, el tutor y los asesores de Estilos de Aprendizaje.

La experiencia del diagnóstico realizada en algunas aulas piloto sirve de experiencia para llevar adelante el diagnóstico en el resto de clases, de forma similar a lo que se hizo en el primer grupo seleccionado de clases, siempre con el apoyo y supervisión de los asesores de Estilos de Aprendizaje y con las reuniones de análisis y planificación, como se realizó en el primer grupo.

Los profesores explican el tema de los Estilos de Aprendizaje a los estudiantes. Los alumnos deberían comprender que cada uno tenemos nuestro estilo preferente de aprender, un estilo que, posiblemente, es distinto del estilo que tienen sus compañeros o su madre, su padre, sus hermanos o sus amigos. Cada estudiante tiene sus puntos fuertes, pero diferentes a los puntos fuertes de otros compañeros. Los Estilos de Aprendizaje se basan en reacciones complejas entre la fisiología personal, los sentimientos, las rutinas, las experiencias vividas, las estrategias de aprendizaje de los profesores que han tenido. Para diagnosticar su Estilo de Aprendizaje se utilizará un instrumento/cuestionario que hayamos seleccionado al que hay que responder con honestidad y sin engañarse. No es un test de inteligencia.

Conviene que todos los estudiantes tengan muy claras algunas reglas fundamentales para que la metodología de los Estilos de Aprendizaje sea una experiencia positiva para todos los alumnos y no una posibilidad de desorientación. Facilitamos un ejemplo de posibles reglas:

- $\quad$ Su estilo de aprendizaje no debe distraer a ningún compañero.

- Con la metodología de los Estilos de Aprendizaje deben mejorar sus calificaciones. 
- $\quad$ Sus tareas deben completarse en el tiempo asignado.

- $\quad$ Cuando el profesor necesite que atienda a lo que él dice, debe atender inmediatamente.

- La manera con la que trabaja en clase se basa en el diagnóstico de su estilo de aprendizaje y las evaluaciones correspondientes.

El docente planifica sus estrategias de enseñanza atendiendo a los resultados obtenidos en el diagnóstico, contando con el apoyo de los asesores de Estilos de Aprendizaje del Centro. No se trata de "etiquetar" a los estudiantes. Intentamos comprender, por ejemplo, quiénes son los estudiantes con predominio de estilo activo, reflexivo, teórico o pragmático, si se ha utilizado el cuestionario CHAEA, para tener en cuenta sus características en nuestro diseño educativo. Y decimos "predominio" pues todos aprendemos con todos los estilos, aunque podemos tener preferencia por uno o dos. El consejo más general es buscar la pluralidad metodológica y la pluralidad de enfoques de evaluación que permitan el aprendizaje a estudiantes con distintos estilos de aprender.

\section{Evaluar-Supervisar}

Es imprescindible incluir en el proceso un espacio significativo para analizar y supervisar lo que se ha llevado a cabo. Sin este análisis el esfuerzo resultaría incompleto. Es importante esta reflexión para planificar el plan de acción para el curso siguiente, corrigiendo los fallos y mejorando los aciertos.

Llevar a cabo una reunión de profesores y asesores para comentar el proceso de diagnóstico y la mejor manera de aplicar en el aula los resultados obtenidos. Es una excelente ocasión para resolver dudas, enriquecer los procesos de cada aula con la experiencia de los procesos llevados a cabo por otros docentes, planificar los pasos siguientes del proceso y planificar las reuniones con los padres de los estudiantes. En ocasiones se detecta la necesidad de completar la formación de los profesores para reforzar su acción educativa y aumentar la eficacia del proceso.

En una reunión con los padres de los alumnos, en que participan también los asesores de Estilos de Aprendizaje, presentar los resultados del diagnóstico y primeras estrategias llevadas a cabo para aprovechar los datos. Qué actividades y 
recursos hay que añadir en el aula para llegar a alumnos de todos los estilos de aprendizaje y cómo ayudar a cada estudiante a mejorar sus estilos de aprendizaje contando con el apoyo de los padres.

Dedicar una asesoría o tutoría a cada estudiante para analizar los resultados que ha obtenido en su diagnóstico. Se trata de ayudarles para aprender mejor. No hay estilos mejores y estilos peores. Todos tienen sus puntos fuertes y débiles. Cada uno tiene que saber aprovechar sus puntos fuertes y desarrollar los puntos débiles. Tratamos de comprender que en la clase cada compañero tiene su propio estilo de aprendizaje y que las actividades del aula no siempre se ajustan a su estilo sino al estilo de otros compañeros, habrá que tener comprensión y paciencia. Analizar qué aspectos debe desarrollar cada uno para tener más capacidad de aprendizaje.

Realizar otra sesión de formación con los profesores si fuera necesaria contando con los asesores locales o con otros expertos en Estilos de Aprendizaje.

El equipo directivo del Centro y los asesores analizan y supervisan los resultados obtenidos en cada una de las aulas para comprobar hasta dónde se ha llegado en el proceso, cuáles han sido los logros obtenidos, mejorar las debilidades y planificar el próximo curso.

Algunas ideas y sugerencias para tener en cuenta durante este proceso y en las diferentes reuniones con profesores y padres, el equipo directivo del Centro debe:

- Observar, apoyar y alabar a los profesores durante el proceso de la implementación de la metodología de los Estilos de Aprendizaje.

- Compartir éxitos e ideas que se aporten por cualquiera de los miembros de la comunidad educativa.

- Reconocer públicamente y alabar el esfuerzo de los asesores, de los profesores, de la asociación de padres.

- Insistir en que todos los estudiantes de la misma clase aprenden el mismo curriculum. La única diferencia es que cada uno aprende con diferentes recursos adaptados a su estilo de aprendizaje. 
- Facilitar el diagnóstico personal de los estilos de aprendizaje a los administrativos, padres, abuelos... y explicar los resultados. Ayuda a crear un clima en el Centro en el que todos están inmersos en la misma metodología.

- Pedir a los padres que ayuden a sus hijos a crear o buscar sus propios recursos de aprendizaje.

- Animar a los profesores para que sigan experimentando y formándose en las posibilidades pedagógicas de los Estilos de Aprendizaje.

- Crear un grupo de profesores voluntarios y padres que sirvan de apoyo y modelo de acción para otros docentes y padres.

- $\quad$ Proponer a los estudiantes más mayores que ayuden a los estudiantes más pequeños en la mejor manera de aprovechar sus estilos de aprendizaje.

- Recoger datos que puedan servir para preparar comunicaciones y testimonio de acción educativa innovadora en reuniones y Congresos.

- Publicar los resultados del proceso llevado a cabo en los medios de comunicación del Centro, en las redes sociales, en revistas, en periódicos, en Congresos.

- Incluir los testimonios de padres, alumnos y profesores que han participado en el proyecto.

- Preparar carteles y posters con los puntos fuertes de la metodología de los Estilos de Aprendizaje, es un recuerdo y una guía para los estudiantes y una información para los visitantes del Centro.

- Continuar leyendo y estudiando publicaciones sobre los Estilos de Aprendizaje, por ejemplo siguiendo la Revista de Estilos de Aprendizaje, que recoge datos e investigaciones actuales sobre el tema http:// revistaestilosdeaprendizaje.com/index

\section{Consideraciones finales}

En este trabajo, muy sintético y concreto, hemos tratado de presentar un enfoque holístico de la metodología de los Estilos de Aprendizaje en una institución educativa, de forma que en el proyecto participen de forma activa y comprometida 
el equipo directivo, todos los profesores, los alumnos y los padres de los estudiantes y se incluya en el proyecto general del Centro. Este plan es una forma de investigación-acción, es decir, investigamos para que lo que hacemos lo hagamos mejor. No es sencillo, pero sí posible, aunque necesita la colaboración y aporte de todos y diseñar una planificación y un cronograma adecuados a la tarea que se propone.

\section{Referencias}

ALKOOHEJI, L.; AL-HATTAMI, A. Learning style preference among college students. International Education Studies, v. 11, n. 10, p. 50-63, 2018.

ALONSO, C. M. Estilos de Aprendizaje: Análisis y Diagnóstico en Estudiantes Universitarios, Madrid: Editorial Universidad Complutense, 1992.

ALONSO, C. M.; GALLEGO, D. J.; HONEY, P. Los estilos de aprendizaje: Procedimientos de Diagnóstico y Mejora, Bilbao: Mensajero, 2012.

ALNUJAIDI, S. The Difference between EFL Students' Preferred Learning Styles and EFL Teachers' Preferred Teaching Styles in Saudi Arabia, English Language Teaching, v. 12, n. 1, p. 90, 2019.

ALTUN, H. Investigation of High School Students' Geometry Course Achievement According to Their Learning Styles. Higher Education Studies, v. 9, n. 1, 2019

ASLAKSEN, K.; LORAS, H. Matching Instruction with Modality-Specific Learning Style: Effects on Immediate Recall and Working Memory Performance. Education Sciences, v. 9, n. 1, 2019. Doi: 10.3390/educsci9010032.

BIABANI, M.; IZADPANAH, S. The Study of Relationship between Kolb's Learning Styles, Gender and Learning American Slang by Iranian EFL Students. International Journal of Instruction, v. 12, n. 2, p. 517-538, 2019.

DIALNET.unirioja.es Universidad Internacional de la Rioja. EDUCATIONAL Resources Information Center, ERIC, www.eric.ed.gov.

EDWARDS, D. J.; KUPCZYNSKI, L.; GROFF, S. Learning Styles in Problem-Based Learning Environments: Impacts on Student Achievement and Professional Preparation in University Level Physical Therapy Courses, International Journal of Higher Education, v. 8, n. 3, p. 206-213, 2019.

FERNANDES, A. A. La inteligencia emocionaly los estilos de aprendizaje: el modelo SAFEM de elearning/b-learning. Tesis (Doctoral - Universidad Nacional de Educación a Distancia, Madrid, UNED, 2011. 
FLEMING, N. D. The case against learning styles: "There is no evidence", 2012, Disponible en: https://vark-learn.com/wp-content/uploads/2014/08/The-Case-Against-LearningStyles.pdf.

GARCÍA CUÉ, J. L., SANTIZO, J. A., ALONSO C. M. Instrumentos de medición de estilos de aprendizaje, Revista de Estilos de Aprendizaje, v. 2, n. 4, p. 1-23, 2009.

GAPPI, L. L. Relationships between learning style preference and academic performance of students. Journal of Educational Research and Technology, v. 4, n. 2, p. 70-76, 2013.

GHOLAMI, S.; \& BAGHERI, M. Relationship between VAK learning styles and problem solving styles regarding gender and students' fields of study. Journal of Language. Teaching and Research, v. 4, n. 4, p. 700-706, 2013.

GOKALP, M. The effect of students' learning styles to their academic success. Creative Education, v. 4, n. 10, p. 627-632, 2013.

GÜNES, G.; SAHIN, V. The Algorithm of Mathematical Modelling for Learning Styles of Pre-School Children. Education, v. 4, n. 3, p. 277-292, 2019.

HUSSMAN, P. \& DEAN O'LOUGHLIN, V. Another nail in the coffin for learning styles? Disparities Among undergraduate anatomy students' study strategies, class performance, and reported VARK learning styles. Anatomical sciences education, v. 12, n. 1, p. 6-19, 2018.

JENA, A.K., \& CHAKRABORTY. Epistemological beliefs: Its relationship with learning styles, learning approaches, and achievement. Asian Pacific, Journal of Education, Arts, and Sciences, v. 5, n. 1, p. 60-70, 2018.

KAMISLI, H.; ÖZONUR, M. Students' Learning Styles in Vocational Education. International Journal of Curriculum and Instruction, v. 11, n. 1, p. 209-220, 2019.

KIRSCHNER, P. Stop propagating the learning styles myth. Computers \& Education, v. 106, n. 1, p. 166-171, 2017.

LI, Y., MEDWELL, J., WRAY, D. Learning styles: A review of validity and usefulness. Journal of Education and Training Studies, v. 4, n. 10, p. 90-94, 2016.

BARROS, D. M. V. Estilos de aprendizaje y medios didácticos en contextos virtuales, Tesis (Doctoral en Educación) - Universidad Nacional de Educación a Distancia, Madrid, UNED, 2011.

NEWTON, P. (2015). The higher learning styles myth is thriving in higher education. Psychology, v. 6, p. 1-5, 1908.

REVISTA de Estilos de Aprendizaje, ISSN-e 1988-8996. Disponible en: <http:// revistaestilosdeaprendizaje.com/>. Acceso en: 15 ene. 2020.

SENER, S., \& COKCALISKAN, A. An investigation between multiple intelligences and learning styles. Journal of Education and Training Studies, v. 6, n. 2, p. 125-132, 2018. 
SHARP, J. G.; BOWKER, R. BYRNE, J. VAK or VAK-ous? Towards the trivialization of learning and the death of scholarship. Research Papers in Education, v. 23, n. 3, p. 293-314, 2008.

SOIFERMAN, K. (2019) The Debate Rages On: Learning Styles Factor Myth Winnipeg: University of Winnipeg ED595102 Teseo. Tesis (Doctorales) - University of Winnipeg, 2019.

WININGER, St. R.; et alii. Prevalence of Learning Styles in Educational Psychology and Introduction to Education Textbooks: A Content Analysis, Psychology Learning and Teaching, n. 9, 2019. Doi: https://doi.org/10.1177/1475725719830301.

RECEBIDO: $11 / 02 / 2019$

APROVADO: $22 / 02 / 2020$

RECEIVED: 02/11/2019

APPROVED: 22/02/2019

RECIBIDO: $11 / 02 / 2019$

APROBADO: 22/02/2019 